\title{
De la experiencia de los agroquímicos a los incipientes desafíos de los nano-agroquímicos: riesgos manufacturados y derecho a un ambiente sano en Argentina
}

\section{From the experience of agrochemicals to the incipient challenges of nano-agrochemicals: the manufactured risks and the right to a healthy environment in Argentina}

\section{Valeria Berros ${ }^{1}$ \\ CONICET-Universidad Nacional del Litoral (Argentina)}

Rodrigo Peiteado ${ }^{2}$

Universidad Nacional del Litoral (Argentina)

Recibido: 27-10-14

Aprobado: 05-01-15

\section{Resumen}

El presente trabajo posee por objetivo iluminar, a partir de la problemática de la exposición a productos agroquímicos, la intersección entre el problema de los riesgos manufacturados y la afectación del derecho humano a un ambiente sano en Argentina. Con ese fin se propone, en una primera parte, analizar el proceso

\footnotetext{
${ }^{1}$ (vberros@fcjs.unl.edu.ar). María Valeria Berros, Doctora en Derecho, Becaria Postdoctoral de CONICET, Docente de la Facultad de Ciencias Jurídicas y Sociales de la Universidad Nacional del Litoral, Investigadora del Grupo Responsable del Proyecto de Investigación: "Codex humano: normas, tecnologías y programas para el gobierno de lo vivo" perteneciente al PACT "Codex global. Regulación en institucionalidad global de lo vivo". Publicaciones recientes: Entre el sumak kawsay y la "vida en armonía con la naturaleza": disputas en la circulación y traducción de perspectivas respecto de la regulación de la cuestión ecológica en el espacio global en coautoría con Victoria Haidar, Revista Theomai, 2014, Principe de non régression au Panamá, Revue Juridique de l'environnement, Volumen 2, Strasbourg, 2014; Regresiones normativas en materia de agro-tóxicos, Informe Ambiental Anual Fundación Ambiente y Recursos Naturales, Buenos Aires, 2014; "Observaciones sobre el principio precautorio en Argentina”, Revista Catalana de Dret Ambiental, Vol. IV. Núm. 2, Tarragona. 2013.

2 (rodrigopei_92@hotmail.com). Rodrigo Peiteado, Becario de Iniciación a la Investigación de la Facultad de Ciencias Jurídicas y Sociales de la Universidad Nacional del Litoral en el marco del Proyecto de Investigación: "Codex humano: normas, tecnologías y programas para el gobierno de lo vivo" perteneciente al PACT "Codex global. Regulación en institucionalidad global de lo vivo". Publicaciones recientes: Gonzalo Bailo y Rodrigo Peiteado, Controversias que plantean las nanotecnologías en el campo jurídico. Aproximaciones desde el derecho comparado: desafios para la tutela de derechos fundamentales individuales y colectivos de incertidumbre, XV Congreso Nacional y V Latinoamericano de Sociología Jurídica. Rosario, 2014.
} 
de judicialización de conflictos que se viene desarrollando sobre este tema a los fines de elucidar el rol que ha tenido el argumento de los derechos humanos en la toma de decisiones judiciales. En la segunda parte, y de cara a los recientes desarrollos en materia de nano-agroquímicos, se intenta construir una reflexión que permita enlazar los interrogantes y desafíos en materia de derechos humanos que dejó planteada la conflictividad en materia de agro-químicos con esta reciente innovación técnica basada en el desarrollo nanotecnológico.

Palabras-clave: derechos humanos, derecho ambiental, agroquímicos, nano-agroquímicos.

\begin{abstract}
The objective of this work is to illuminate -from the problematic of the exposure to agrochemical products- the intersection between the problem of the manufactured risks and the affectation of the human right to a healthy environment in Argentina. We purpose to analyze the process of conflict's judicialization which is being developed about this topic in order to elucidate the role that the argument of humans right has had in the judicial decision-making. In the second part we try to build a reflection which allows linking questions and challenges in the matter of human rights which has raised the conflict in the matter of agrochemicals with this recent technical innovation based on the development about nanotechnology.
\end{abstract}

Key-words: Human Rights, Environmental Law, Agrochemical, Nanoagrochemicals

\title{
I. Introducción
}

El objeto del presente trabajo consiste en realizar un aporte en la intersección entre el derecho humano a un ambiente sano y el desarrollo de nuevas tecnologías aplicadas al sector de producción agraria en Argentina. Se parte del análisis de la problemática de la expansión del uso de agroquímicos en este país así como de la emergente discusión sobre la aplicabilidad de nano-agroquímicos lo que, a la vez que reedita algunos debates vinculados con la tutela de los derechos humanos y el ambiente, pone a consideración nuevas aristas e interroga acerca de las lecciones que podrían extraerse de la experiencia ya transitada.

En Argentina, desde el año 1996, se ha autorizado el cultivo de organismos genéticamente modificados, lo que dio lugar a un considerable aumento de la cantidad de agroquímicos utilizados cuyas consecuencias perjudiciales, tanto respecto del ambiente como de la salud humana, no tardaron en ser denunciadas. 
Las respuestas desde la ciencia en relación a este tema no son homogéneas; por el contrario, existe al respecto un panorama controversial en el campo científico que se articula con otros datos muy relevantes provenientes de profesionales de la salud, organizaciones no gubernamentales y de los mismos afectados por las fumigaciones y cuya importancia fue central para ubicar el tema entre los contenidos que configuran la agenda pública y que, además, interrogan a la ciencia como único espacio para la producción se saberes. Los focos de discusión iluminan aspectos tanto vinculados al ambiente como a la salud.

En el primer caso, se hace referencia a la potencialidad que el uso de este tipo de productos posee en la modificación de los ecosistemas provocando pérdida de biodiversidad y de otras culturas agrícolas, a lo que se suma la contaminación del suelo y del agua.

En el segundo, desde diferentes instituciones se han efectuado estudios en relación a las alteraciones en el sistema reproductivo, la posibilidad de que se constituyan como agentes generadores de acciones mutagénicas o carcinogénicas, el grado de toxicidad de estas sustancias, la provocación de enfermedades dermatológicas y respiratorias, entre otras.

Con claridad se advierte que este tema conjuga tanto la tutela del derecho humano a un ambiente sano como la protección de la naturaleza y diversidad biológica per se. En este contexto, y sobre el problema del uso de agroquímicos, comenzó a desarrollarse hace casi una década un proceso de judicialización de conflictos que pueden ser distinguidos, a fines analíticos, según el contenido de lo demandado por los actores: i) grupos de vecinos que reclaman la paralización de fumigaciones por incumplimientos de distancias legalmente establecidas ${ }^{3}$; ii) empresas que solicitan la declaración de inconstitucionalidad de regulaciones que propenden a limitar o prohibir el uso de agroquímicos; grupos de vecinos que requieren se declare la inconstitucionalidad de normas locales consideradas regresivas respecto de la normativa anterior aplicable ${ }^{4}$.

Se introduce así en la instancia judicial este tema en el que el aspecto medular es el estado de controversia científica sobre los posibles daños que generarían a

\footnotetext{
${ }^{3}$ Entre estos casos se destacan: Arrocera San Carlos SRL y Arrocera Cancha Larga S.A E/A Ferrau, Marco Antonio y otros c/ Municipalidad de Las Palmas y otros s/medida cautelar, Sala I de la Cámara Civil y Comercial de Apelaciones de la ciudad de Resistencia, 21.02.2011; Cavigliano Peralta, Viviana y otros c/ Municipalidad de San Jorge y otros s/ amparo, Juzgado de Primera Instancia de Distrito en lo Civil, Comercial y Laboral de San Jorge, Provincia de Santa Fe, del 16.03. 2009, Sala II de la Cámara en lo Civil y Comercial de la ciudad de Santa Fe del 09.12. 2009, Juzgado de Primera Instancia de Distrito en lo Civil, Comercial y Laboral de San Jorge del 21.02.2011, Sentencia del 19.04.12 y aclaratoria del 16.05.12 de la Sala II de la Cámara en lo Civil y Comercial de la ciudad de Santa Fe; Monsalvo, Cristina y otros c/ Delaunay, Jorge s/ amparo, Corte Suprema de Justicia de la Provincia de Buenos Aires, 08.08.2012.

${ }^{4}$ En este conjunto de casos se destacan: Chañar Bonito S.A. c/ Municipio de Mendiolaza s/ amparo, Supremo Tribunal de Justicia de la Provincia de Córdoba, 18.09.2007; Speedagro SRL c/ Comuna de Arequito s/ medida cautelar, Sala II de la Cámara en lo Contencioso Administrativo de Rosario 03.10.2011; Picorelli, Jorge Omar y otros c/ Municipalidad de General Pueyrredón s/ inconstitucionalidad Ord. $N^{\circ} 21.296$, Corte Suprema de Justicia de Buenos Aires, 24.09.2014.
} 
la salud humana y al ambiente. En este camino, el argumento de la afectación de los derechos humanos al interior de las decisiones judiciales permanece aún poco atendido aunque, es cierto, en algunos recientes casos se lo ha comenzado a desarrollar en dos sentidos: i) como refuerzo explícito en la línea argumental de tutela del derecho a un ambiente sano, ii) mediante el uso del principio de no regresión ambiental que ha sido construido por medio de un proceso de traducción de una herramienta propia de los derechos humanos - y contracara del principio de progresividad - hacia el campo de la protección del ambiente.

A este recorrido se suma, desde hace poco tiempo, el debate sobre los nano-agroquímicos que puede ser observado como una oportunidad para revisar el tránsito sobre la experiencia ya recorrida con los agroquímicos. Aquí también se advierten vacilantes aproximaciones que, desde el ámbito científico, se construyen sobre las consecuencias potencialmente asociables al desarrollo de nano-agroquímicos, producidos a partir de la aplicación de la tecnología nano a los agroquímicos, y con el fin de aplicarlo a la producción agraria.

Los desarrollos tecnológicos que intentan aumentar la productividad agraria desde diferentes estrategias plantean varios desafíos en torno a cómo se decide sobre este tipo de riesgos manufacturados y controvertidos, cuáles son los conocimientos valiosos a considerar, cómo operan allí los argumentos relativos a la protección de los derechos humanos y de la naturaleza. En otros términos, suponen importantes desafíos de regulación y construcción institucional que canalicen una toma de decisiones colectiva, informada y que atienda a las consecuencias dañosas que se articulan con estos agentes.

Estos interrogantes y reflexiones permean el presente trabajo. En lo que sigue se propone, en el apartado II, una breve e introductoria caracterización sobre la expansión del uso de agroquímicos en Argentina. En la tercera parte se presentan los planteos judiciales circunscriptos a las sentencias en que sus líneas argumentales han comenzado, recientemente, a asignar espacio al argumento de la tutela de los derechos humanos así como a algunas herramientas inspiradas en este campo del derecho como el principio de no retroceso, traducido desde los derechos humanos hacia el derecho ambiental, para la resolución de conflictos. En el apartado IV se construyen algunas preguntas sobre la discutida introducción de nano-agroquímicos a la producción agraria y los desafíos que allí se identifican. Por último, se enumeran algunas agendas de construcción jurídico-institucional que podrían ser relevantes a los efectos de edificar un sistema de toma de decisiones cuyo eje medular consista en la tutela del derecho humano a un ambiente sano, así como en la protección de la naturaleza frente a riesgos controvertidos, en el que se integre tanto la ciencia como otras áreas de producción de conocimientos. 


\section{Agroquímicos: expansión y principal normativa argentina}

En Argentina, el incremento del uso de agroquímicos, se relaciona con la autorización que desde la Secretaría de Agricultura se efectuara en 1996 sobre el cultivo de semillas modificadas genéticamente y, en particular, de soja modificada genéticamente tolerante al glifosato ${ }^{5}$.

Esta habilitación se une, además, a la coyuntura internacional de aumento de los precios internacionales de los agro-alimentos lo que coadyuvó a la veloz expansión del denominado 'proceso de sojización'. El mismo, por una parte, modifica el modo de cultivo de la región agraria y, por la otra, la amplía hacia zonas en las que no se cultivaba con antelación avanzándose así sobre regiones boscosas y montes, lo que se tradujo en mayores niveles de conflictividad por el uso del territorio.

Con el correr de algunos años, no tardaron en aparecer movilizaciones sociales de diferente tipo, grupos de vecinos, organizaciones no gubernamentales, profesionales de la salud y miembros del sistema científico, que denunciaron y trabajaron por demostrar los negativos efectos que este nuevo modelo de producción terminaba por generar. Un acento especial parece haber sido dispuesto sobre el glifosato, herbicida no selectivo creado en la década del sesenta imbricado en la llamada "revolución verde" que, desde algunos años antes, había propiciado la inserción del uso intensivo de insumos químicos a los efectos de eliminar malezas y aumentar la productividad y, por tanto, la rentabilidad.

Se verifica aquí la introducción de un "riesgo manufacturado" al medio y sus consecuencias perjudiciales, en principio, no son visibilizadas. Este estado de cosas, sin embargo, en poco tiempo sufre una metamorfosis que pone en diálogo distintas cuestiones: (i) la aparición de estudios e investigaciones sobre la toxicicidad de la sustancia ${ }^{7}$, (ii) el surgimiento de micro-movilizaciones

5 Esta autorización se expresa en la Resolución $N^{\circ} 167$ del año 1996 de la Secretaría de Agricultura, Ganadería, Pesca y Alimentos de la Nación que se configura como la primera de una serie de resoluciones de similar naturaleza.

${ }^{6}$ El sociólogo alemán Ulrich Beck es quien hace referencia a la idea de riesgos manufacturados o fabricados por el hombre cuando alude a las consecuencias no buscadas del desarrollo científicotecnológico lo que se articula con su planteo acerca de la denominada "sociedad del riesgo" que presenta las características a partir de las cuales se consolida lo que él denomina "segunda modernidad" lo que fue plasmado en "La sociedad del riesgo. hacia una nueva modernidad" (1998), Barcelona, Paidos. En coautoría con Christoph Lau, Beck reflexiona sobre la agenda de investigación sociológica contemporánea en "Second modernity as a research agenda: theoretical and empirical explorations in the "meta change"'of modern society”, "The British Journal of Sociology", Volume 56 Number 4, December 1994.

${ }^{7}$ En Argentina se destaca, verbigracia, el equipo liderado por Argelia Lenardón en el Laboratorio de Medioambiente del INTEC, UNL-CONICET en Santa Fe que ha estudiado la presencia de organoclorados en leche materna en un grupo de mujeres del norte de esa localidad así como los trabajos que el mismo grupo realizó sobre el problema en medios acuosos estáticos y en vertebrados silvestres del litoral fluvial; las investigaciones eco-toxicológicas de Rafael Lajmanovich en el Laboratorio de Ecotoxicología de la Facultad de Bioquímica y Ciencias Biológicas de la UNL en Santa Fe; los estudios efectuados por Amalia Dellamea en la Facultad de Farmacia y Bioquímica de la UBA sobre la existencia de residuos de agroquímicos en productos lácteos; las indagaciones sobre las consecuencias toxicológicas del uso de agroquímicos realizados por Jorge Kaczewer investigador 
sociales en distintas localidades y de organizaciones no gubernamentales de índole ambiental o rural que comienzan a atender a la problemática ${ }^{8}$, (iii) las alertas emitidas por profesionales de la salud - particularmente médicos rurales - advirtiendo sobre la temática? .

Las investigaciones se vienen desarrollando desde hace varios años y las conclusiones a las que arriban han sido objeto de críticas y respuestas ya que, en el otro polo de la controversia, existe también producción de conocimiento en sentido contrario, considerando que los agroquímicos no presentarían mayores riesgos. De modo concomitante, las movilizaciones locales cobran mayor repercusión pública y quienes durante años anteriores habían emitido alertas sobre el tema terminan de visibilizarse. Asimismo, desde hace ya varios años se desenvuelven numerosos encuentros en una gran cantidad de localidades en la región agrícola en los que se escucha a afectados, profesionales de la salud y a integrantes de diferentes equipos de investigación que han trabajado en la temática desde hace mucho tiempo.

Por su parte, a los efectos de analizar cómo se encuentra regulado el problema de los agroquímicos, se debe efectuar una cartografía que pone en relación diferentes normativas: (i) La legislación nacional que ha creado instituciones competentes en la temática; (ii) Las normas provinciales y locales que determinan las condiciones de utilización de los agro-químicos en el plano territorial; (iii) Las regulaciones que de modo tangencial y fragmentario hacen referencia al tema.

En 1996 no sólo se autoriza el cultivo de semillas modificadas genéticamente sino que, mediante el Decreto 1585/1996, se instituye el Servicio

de la UBA y, a la vez, miembro de la organización no gubernamental Grupo de Reflexión Rural; las conclusiones construidas por el médico Alejandro Oliva de la Unidad de Andrología del Hospital Italiano de Rosario que analizó el efecto de esta sustancia en el sistema reproductivo masculino y el riesgo de empobrecimiento del nivel de esperma; las investigaciones sobre embriología molecular realizadas por Andrés Carrasco, quien fuera Director del Laboratorio de Embriología Molecular de la Universidad de Buenos Aires e Investigador de CONICET y cuyos aportes fueron medulares para las luchas contra este modelo de producción agraria.

${ }^{8}$ En Argentina existen numerosos focos de movilización social en relación a este tema, algunos de los que han tenido mayor repercusión pública fueron las "madres del Barrio Ituzaingó" en Córdoba, la movilización en la ciudad de San Francisco en la misma provincia que condujo a la sanción de la Ordenanza $\mathrm{N}^{\circ} 5531$ que determina una "zona de resguardo ambiental" de 500 metros en la que no se pueden aplicar agroquímicos; el "caso San Jorge" en la localidad de dicho nombre en la Provincia de Santa Fe que, mediante una acción de amparo, logra suspender las fumigaciones por un período de tiempo aún vigente y también obtiene la sanción de una ordenanza en junio de 2010 en la que se fija una zona de 100 metros - contados desde el límite del ejido urbano - libre de productos fitosanitarios. Respecto de las organizaciones no gubernamentales que poseen especial injerencia en este tema desde hace años, se destaca la campaña "Paren de Fumigarnos" que nuclea organizaciones de diferentes puntos del país.

${ }^{9}$ Aquí se destaca a Raúl Lucero, bioquímico que realizó un estudio en relación a la genotoxicidad en Chaco; el médico pediatra Rodolfo Páramo que comenzó a observar el problema en razón de la cantidad de nacimientos con malformaciones en Malabrigo en Santa Fe; el médico rural Darío Gianfelici que se comenzó a preocupar por el tema por las mismas razones y las observaciones que efectuó sobre los cambios en el ecosistema del litoral. 
Nacional de Sanidad y Calidad Agroalimentaria (SENASA) como organismo descentralizado de la Secretaría de Agricultura, Ganadería, Pesca y Alimentación - que ha quedado hoy reconfigurada dentro del organigrama del Ministerio de Agricultura, Ganadería y Pesca de la Nación -y cuya responsabilidad consiste en "ejecutar las politicas nacionales en materia de sanidad y calidad animal $y$ vegetal, verificando el cumplimiento de la normativa vigente en la materia. Asimismo, entenderá en la fiscalización de la calidad agroalimentaria, asegurando la aplicación del Código Alimentario Argentino para aquellos productos del área de su competencia” (Art. 2 Decreto $\mathrm{N}^{\circ}$ 1585/96).

De esta manera, SENASA se constituye como la autoridad competente a nivel nacional en cuanto a agroquímicos de uso industrial y agrícola y asume el control de la elaboración, fraccionamiento, distribución, tenencia y expendio de este tipo de productos. Dentro de su organigrama se conforma la Coordinación General de Agroquímicos y Biológicos que es competente para la inscripción de productos fitosanitarios mediante una serie de procedimientos pautados reglamentariamente.

Hasta aquí la regulación de los agroquímicos posee fuerte raigambre comercial, delineando los parámetros pertinentes para su tratamiento como producto. Este es el andamiaje institucional nacional que propone los pilares básicos para la gestión de los productos fitosanitarios desde una perspectiva que focaliza en su costado de producto comercializable y las condiciones para su inserción en el mercado.

En 2003, desde una lógica que podría articularse con una "renovación" de la mirada sobre el problema de los agroquímicos, se crea el Sistema Federal de Fiscalización de Agroquímicos y Biológicos ${ }^{10}$ que, además de focalizar en la fitosanidad de los productos, introduce la noción de seguridad alimentaria, el velar por la salud poblacional y el cuidado del ambiente, y postula la generación de un sistema de trazabilidad de productos a los fines de alcanzar tales objetivos. Este sistema se integra por una serie de instituciones - tanto de carácter público como privado - en "interacción recíproca" con el objeto de realizar un control sobre todo el proceso de producción de fitosanitarios destacando la necesidad de prevenir daños y mejorar la productividad de modo sustentable.

Por su parte, en el ámbito provincial y local existen numerosas regulaciones. En la gran mayoría de las provincias se encuentran leyes sobre la materia que se encargan de regular aspectos referidos a la utilización de los productos y sus condiciones de empleo, dentro de las cuales un dato que adquiere especial relevancia son las modalidades de aplicación de agroquímicos y las zonas en que se lo permite. Es decir, a qué cantidad de metros desde "lo urbano" se pueden utilizar agroquímicos lo que, generalmente, se pauta de distinto modo según la aplicación sea aérea o terrestre.

${ }^{10}$ Este organismo fue creado por Resolución 500/2003 de SENASA y luego modificado por Disposición 119/2007.

Araucaria. Revista Iberoamericana de Filosofía, Política y Humanidades, año 17, $\mathrm{n}^{\circ} 33$. Primer semestre de 2015. Pp. 229-251. ISSN 1575-6823 e-ISSN 2340-2199 doi: 10.12795/araucaria.2015.i33.10 
Por último, existen algunas regulaciones que tangencialmente hacen referencia al problema desde diversas perspectivas como la Ley 20.418/73 sobre tolerancias y límites administrativos de residuos de agroquímicos o la Ley 24.051/92 de residuos peligrosos en cuyo primer Anexo se mencionan los desechos resultantes de la producción, preparación y utilización de biocidas y productos fitosanitarios.

Si bien no aparece una clara vinculación entre este plexo regulatorio, la tutela ambiental y la protección de los derechos humanos, desde 1994, la Constitución Nacional en el apartado que incorpora nuevos derechos y garantías, en el artículo 41, hace referencia al derecho a un ambiente sano en sintonía con el proceso de reconocimiento de este derecho en las reformas constitucionales latinoamericanas de entonces.

\section{Derechos humanos: argumento emergente en recientes sentencias judiciales}

En este contexto, si bien hace casi dos décadas que Argentina se encuentra desarrollando su producción agraria en base al trípode organismos genéticamente modificados, agroquímicos y siembra directa, hace menos tiempo que tribunales de diferentes provincias y fueros han tenido que decidir sobre este tema.

Ante la instancia jurisdiccional, se han presentado dos cuestiones a resolver: (i) Incumplimientos de distancias legalmente establecidas para la fumigación, lo que es sostenido por grupos de vecinos perjudicados; (ii) Inconstitucionalidad de normas locales que prohíben o limitan el uso de agroquímicos. Este tipo de demandas se presenta por empresas que se consideran perjudicadas o por parte de grupos y vecinos que catalogan como regresiva alguna modificación normativa.

De entre las sentencias que han resuelto sobre ambos temas encontramos, por una parte, un conjunto de demandas rechazadas por cuestiones de forma ante la poca plasticidad que poseen las vías de tutela inhibitoria disponibles y, por la otra, un grupo en el que se resuelve a partir de argumentos que suelen oscilar entre la aplicación del principio precautorio y la tutela del derecho al ambiente que reconoce la Constitución Nacional en su artículo 41.

En estas últimas sentencias, sin embargo, es difícil encontrar en las líneas argumentales que jueces y juezas explícitamente aludan a la protección de los derechos humanos y al sistema de protección que se ha consolidado con ese fin. Por el contrario, se trata este de un tema que comienza a aparecer, de manera enfática y expresa, desde hace poco tiempo en el texto de sentencias judiciales específicas sobre el tema de agroquímicos que serán analizadas a continuación. 
La primera de ellas se ubica dentro del grupo (i) que alude a la paralización de fumigaciones ilegales y la segunda puede ser ubicada en el conjunto (ii) que se focaliza en el análisis de constitucionalidad de normas.

De entre el conjunto de sentencias que resuelven sobre fumigaciones ilegalmente realizadas, el caso que aquí comentamos comienza a sustanciarse a partir de la interposición de una acción de amparo en 2008 ante la materialización de fumigaciones a poca distancia de la zona urbana y a muy escasos metros de la casa de la familia Monsalvo, que son quienes inician esta acción, los padres por derecho propio y en representación de sus hijos menores.

Solicitaron judicialmente: (i) limitar a doscientos metros desde la zona urbana la realización de fumigaciones lo que afectaría una franja de entre 8 y 9 hectáreas de la explotación agrícola del demandado e importaría una aplicación extensiva a las fumigaciones terrestres de lo previsto por el art. 38 del Decreto Reglamentario $\mathrm{N}^{\circ} 499 / 01$ para las aéreas, (ii) implementar un cerco vivo a los fines de aminorar los riesgos provenientes de la exposición a este tipo de tóxicos.

En la primera instancia judicial de la causa Monsalvo, María Cristina y otro c/ Delaunay, Jorge Enrique s/amparo, resuelta por el Juzgado Correccional Nro 4 de Mercedes de la Provincia de Buenos Aires, se rechaza el amparo. El fundamento central remarca la no acreditación de daño alguno en la salud de los actores lo que se constituye, por un lado, como llamativo debido a que lo solicitado en el amparo no se relaciona con la reparación del daño sino con el cese de la actividad y, por otro, deja al descubierto una mirada respecto de la problemática muy imbricada en la tesis tradicional que postula el sistema del derecho de daños que requiere de certeza para su funcionamiento, lo cual no se condice con la hipótesis de hecho respecto de la cual se debe resolver. De este modo, la decisión opaca la controversia científica que existe sobre el agente contaminante, que se plantea en términos de posibilidades y no de certezas, todo lo cual ha recibido su traducción en el ámbito del derecho ambiental en el que el daño es repensado a la luz de los principios preventivo y precautorio que lo informan.

Este fallo fue apelado y en la segunda instancia judicial se confirma el decisorio desde un desconocimiento del principio precautorio y una mirada desarticulada de la prueba producida. El principio de precaución ha sido aplicado en numerosas sentencias judiciales sobre este tema y aparece de modo más o menos cardinal en las líneas argumentales de los decisorios. En el caso argentino, el mismo ha sido incorporado por la llamada Ley General del Ambiente, Ley $\mathrm{N}^{\circ} 25.675 / 02$, como uno de los principios de política ambiental nacional y se conceptúa del siguiente modo: "Cuando haya peligro de daño grave o irreversible la ausencia de información o certeza científica no deberá utilizarse como razón para postergar la adopción de medidas eficaces, en función de los costos, para impedir la degradación del medio ambiente” (Art. 4 Ley N²5.675) 
Este principio abre en simultáneo dos agendas. La primera es la revisión del vínculo entre derecho y ciencias, poniendo de relieve que esta última no reviste la mentada homogeneidad y objetividad a través de las que se "presentó" modernamente ${ }^{11}$. La segunda es el espacio que se asigna a otras formas de conocimiento que han sido hasta el momento soslayadas pero que, actualmente, revisten gran importancia. Las experiencias de vida, las biografías y la cotidianeidad se configuran como muy relevantes a los efectos de trabajar con problemáticas de riesgos no acabadamente conocidos y la mirada y canalización respecto de estos saberes "legos" requiere una revalorización ${ }^{12}$.

En la sentencia de segunda instancia de la Sala I de la Cámara de Apelación y Garantías en lo Penal, Mercedes, Provincia de Buenos Aires, el principio de precaución sólo aparece de modo muy tangencial apropiándose de las afirmaciones vertidas en la expresión de agravios por parte de la actora:

"la esencia del principio de precaución es que la sociedad no puede esperar
hasta que se conozcan todas las respuestas, antes de tomar medidas que protejan
la salud humana o el medio ambiente de un daño potencial y agregó que la pre-
caución es necesaria cuando dos circunstancias se presentan a la vez: a) falta de
certidumbre científica y b) amenaza de daño al ambiente o a la salud humana"13

pero, más allá de ello, no se resuelve el caso como una hipótesis de precaución. Por el contrario, la sentencia da cuenta de una mirada superficial sobre el principio que no indaga en sus implicancias prácticas, de un desconocimiento e invisibilización de la conflictividad social que se configura como contexto del caso y de un confinamiento del tema hacia la normativa aplicable sin poner en relación dichas normas con la problemática de riesgo que las mismas traducen.

El tribunal, luego de efectuar un recorrido por la normativa que considera aplicable al caso, refiriéndose a normas ambientales de índole nacional que reconoce el derecho a un ambiente sano (art. $41 \mathrm{CN}$ ), provincial (art. 28 de la

${ }^{11}$ Sobre el vínculo entre derecho y ciencia puede consultarse: José Esteve Pardo, El desconcierto del Leviatán. Política y derecho ante las incertidumbres de la ciencia, Marcial Pons, Madrid, 2009; José Esteve Pardo, Técnica, riesgo y derecho. Tratamiento del riesgo tecnológico en el Derecho Ambiental, Ariel. Barcelona, 1999; ChristineNoiville, Du bon gouvernement des risques. Le droit et la question du "risque acceptable ", Paris, Presses Universitaires de France, 2003; Boaventura de Sousa Santos, Crítica de La razón indolente. Contra el desperdicio de la experiencia, Bilbao, Editorial Desclée de Brouwer, 2003; Boaventura de Sousa Santos, Um discurso sobre as ciências, Porto, Ediciones Afrontamento, 2002.

${ }_{12}$ En este sentido, los aportes desde los estudios sociales de la ciencia y la tecnología son medulares y deberían permitir repensar muchas de las instituciones y regulaciones jurídicas en las que la ciencia es considerada como único canal de producción de saberes. Para una introducción sobre este campo disciplinar puede consultarse: Dominique Pestre, Introduction aux sciences studies, Paris, La Découverte, 2006.

${ }^{13}$ Fragmento sentencia "Monsalvo, María Cristina y otro c/Delaunay, Jorge Enrique s/amparo" Sala I de la Cámara de Apelación y Garantías en lo Penal, Mercedes, Provincia de Buenos Aires, 16.05.2010. 
Constitución de la Provincia de Buenos Aires y Ley 11.723/95 de la provincia de Buenos Aires), relativas al uso de productos fitosanitarios (Ley Provincial sobre agroquímicos N$^{\circ} 10.699 / 1998$ y su Decreto Reglamentario, Ordenanza N¹690 del Partido de Alberti de la Provincia de Buenos Aires), afirma que debe verificarse si la actividad desarrollada resulta adecuada a la normativa reglamentaria o no $\mathrm{y}$, en su caso, si afectó o puede afectar en modo alguno la vida y la salud de las personas involucradas, cuestión que luego no es retomada en este sentido.

Asimismo, es llamativo que se haga expresa referencia a que la instalación del barrio allí es posterior a la explotación agropecuaria y que "la agricultura es una práctica que en nuestro país es de las más desarrolladas e influyentes del mundo con todo lo que ello implica, no se advierte que las técnicas denunciadas resulten arbitrarias o ilegales de modo manifiesto"14. Pareciera que la tradición agropecuaria argentina "con todo lo que ello implica" puede convertirse en justificación para fumigar a escasos metros de la vivienda de una familia o de un barrio de una localidad que se instaló allí, claro, luego de haberse consolidado el modelo agroexportador hacia fines del siglo XIX.

Sin embargo, desde entonces, no sólo se ha modificado el acento puesto en la protección del ambiente y la salud humana sino, también, cambió el modo de producción introduciendo "nuevos riesgos", "riesgos manufacturados" en pos de aumentar la productividad, conforme la propuesta de un modelo de producción capitalista global. Esto último aparece silenciado aún cuando existen elementos bastante claros que son incorporados por el propio tribunal al texto de la sentencia y luego no son recuperados. A su vez, se desconoce la controversia que caracteriza esta hipótesis y se considera que de la prueba producida no pudo acreditarse ninguna lesión en la salud de los actores lo que demuestra un razonamiento en términos de certeza científica poco articulable con la controversia que subyace a esta temática.

La Corte Suprema de Justicia de Buenos Aires, el 8 de agosto de 2012, en la última instancia, adopta una resolución que es relevante respecto del objetivo de este trabajo, dado que allí se enfatiza el argumento de derechos humanos. Esta resolución se configura como una de las huellas emergentes y explícitas sobre la necesaria tutela de los derechos humanos en relación al problema de los agroquímicos. Aquí la Corte revoca la sentencia anterior y resuelve a favor de los actores ordenando se prohíban las fumigaciones por fuera de los parámetros legalmente establecidos, a la vez que introduce una referencia al caso como supuesto en que se vulnera el sistema legal aplicable tendiente a la protección de los derechos humanos, lo que se advierte como un elemento novedoso en el campo de las decisiones judiciales sobre este tema.

${ }^{14}$ Fragmento sentencia "Monsalvo, María Cristina y otro c/Delaunay, Jorge Enrique s/amparo" Sala I de la Cámara de Apelación y Garantías en lo Penal, Mercedes, Provincia de Buenos Aires, 16.05.2010. 
En esta sentencia no sólo se alude al principio de precaución así como a la particularidad que posee este tipo de amparo como mecanismo de tutela inhibitoria sino que, también, se cita jurisprudencia de la Corte Interamericana de Derechos Humanos a los efectos de reforzar el argumento de la protección del derecho al ambiente sano en tanto derecho humano. Asimismo, agrega que es oportuno recordar que la Corte Interamericana de Derechos Humanos ya ha afirmado que el derecho a la vida es un derecho humano fundamental y prerequisito para el disfrute de todos los demás derechos -dentro de los cuales se identifica el derecho a un ambiente sano- a lo que también añade que esa misma corte internacional ya ha afirmado que los derechos a la vida y a la integridad humana se encuentran directamente vinculados con la salud humana ${ }^{15}$.

Aquí se detecta, entonces, una de las incipientes huellas al interior de las líneas argumentales de la jurisprudencia reciente que se articulan con el plano de la tutela de los derechos humanos en materia de agroquímicos en Argentina.

Otra de las huellas, marcas, que comienza a ingresar en el ámbito de las decisiones sobre este tema se relaciona con el principio de no regresión. El mismo, caro a la tradición de los derechos humanos, recientemente ha sido traducido hacia el ámbito ambiental lo que da cuenta de una interesante relación entre derecho ambiental y derechos humanos más allá del reconocimiento del derecho a un ambiente sano en tanto derecho humano.

En este caso se trata de adaptar un mecanismo, surgido como contracara del principio de progresividad, hacia el ámbito de la protección ambiental ante posibles regresiones. Esta iniciativa de traducción surgió a partir de un grupo de investigadores argentino-franceses de la Universidad Nacional del Litoral y de la Université de Limoges ${ }^{16}$. Su finalidad era construir una herramienta a ser utilizada

\footnotetext{
${ }_{15}$ Afirma expresamente la sentencia: Sobre esa base, resulta oportuno recordar que La Corte Interamericana de Derechos Humanos ha afirmado que "el derecho a la vida es un derecho humano fundamental, cuyo goce es un prerrequisito para el disfrute de todos los demás derechos humanos" (caso Ximenes Lópes, Sentencia del 4 de julio de 2006, Serie C $n^{\circ}$ 149, párr. 124; caso Baldeón García, sentencia de 6 de abril de 2006, Serie $C n^{\circ} 147$, párrs. 82 y 83; caso Comunidad Indigena Sawhoyamaxa, sentencia del 29 de marzo de 2006, Serie C n ${ }^{\circ}$ 146, párrs. 150, 151 y 152; caso de la Masacre de Pueblo Bello, sentencia del 31 de enero de 2006, Serie C no 140, párrs. 119 y 120; caso de la Masacre de Mapiripán, sentencia del 15 de septiembre de 2005, Serie C $n^{\circ} 134$, párr. 232; caso Comunidad Indigena Yakye Axa, sentencia del 17 de junio de 2005. Serie C no 125, párrs. 161 y 162; caso Huilca Tecse, sentencia del 3 de marzo de 2005, Serie C n ${ }^{\circ}$ 121, párrs. 65 y 66; Caso Instituto de Reeducación del Menor, sentencia del 2 de septiembre de 2004, Serie C no 112, párrs. 156 y 158; caso de los Hermanos Gómez Paquiyauri, sentencia del 8 de julio de 2004, Serie C $n^{\circ} 110$, párrs. 128 y 129; caso 19 Comerciantes, sentencia del 5 de julio de 2004, Serie C no 101, párrs. 152 y 153; caso Juan Humberto Sánchez, sentencia del 7 de junio de 2003, Serie C no 99 párr. 110; caso de los Niños de la Calle -Villagrán Morales y otros sentencia del 19 de noviembre de 1999. Serie C $n^{\circ}$ 63, párr. 144); añadiendo que "los derechos a la vida y a la integridad humana se hallan directa e inmediatamente vinculados con la atención de la salud humana” (Corte I.D.H., Caso Albán Cornejo y otros. Vs. Ecuador, sentencia del 22 de noviembre de 2007, Serie C $n^{\circ} 171$, párr. 117). Fragmento sentencia Monsalvo, María Cristina y otro c/Delaunay, Jorge Enrique s/amparo, Corte Suprema de Justicia, Provincia de Buenos Aires, 08.08.2012.

${ }^{16}$ El equipo francés se encuentra integrado por Michel Prieur, Jessica Makowiak, Gérard Monediaire, Hubert Delzangles y Christophe Krolik y el equipo argentino se conforma por Gonzalo 
ante regresiones en los niveles de protección ambiental de diferente tipo, ya sea en la legislación, en las políticas públicas, en la jurisprudencia, etcétera.

Luego de un período de consolidación de sus fundamentos propios, versados en la articulación entre la noción de generaciones futuras y la revisión del concepto de desarrollo ${ }^{17}$, el principio comenzó a circular entre los argumentos de demandas judiciales así como entre los fundamentos de normas legales ${ }^{18}$.

Por el momento, existe una muy reciente sentencia judicial que ha utilizado este principio para decidir en un caso que puede ser ubicado dentro del segundo conjunto de problemas que se trataron judicialmente hasta el momento: la constitucionalidad de normas locales que buscan restringir o prohibir el uso de productos agroquímicos en su territorio.

Esta sentencia es resultado de una demanda presentada por reclamo por inconstitucionalidad de los arts. 19, 23, 27, 28 y 35 de la Ordenanza Municipal del Partido de Gral. Pueyrredón promulgada bajo el N 21.296 en mayo de 2013. Este nuevo cuerpo legal modificó los parámetros que estaban dispuestos por la normativa anterior del año 2008 y estas modificaciones fueron consideradas regresivas dado que eliminan la zona se seguridad que establecía un radio de mil metros a partir del límite de la planta urbana y de grupos poblacionales en el que se encontraba prohibido fumigar; y también disminuye el nivel de protección al aminorar la cantidad de metros respecto de áreas urbanas, cursos de agua y escuelas rurales en los que no se puede fumigar con agroquímicos.

El planteo judicial es realizado por un grupo de vecinos afectados y organizaciones sociales que, entre el conjunto de argumentos que utilizan, remiten a la necesidad de aplicar el principio de no retroceso. El caso, Picorelli, Jorge Omar y otros c/ Municipalidad de General Pueyrredón s/ inconstitucionalidad Ord. $N^{\circ} 21.296$, también llega hasta la tercera instancia

Sozzo, Valeria Berros, Lorena Bianchi y Luciana Sbresso. En conjunto presentaron, en la Convocatoria del año 2010, ante ECOS Sud Francia y el Ministerio Nacional de Ciencia y Tecnología de Argentina, un proyecto de investigación binacional sobre la construcción del principio de no regresión en el derecho ambiental que se ejecutó entre los años 2011 y 2013.

${ }_{17}$ Una primera publicación colectiva del equipo y otros investigadores de diferentes países del mundo que da cuenta de este y otros temas relativos al principio de no regresión: Michel Prieur y Gonzalo Sozzo (Coord.) La non régression en droit de l'environnement, Bruxelles, Bruylant, 2012. Sobre la construcción de este principio en Argentina puede consultarse: María Valeria Berros, "Construyendo el principio de no regresión en el derecho argentino" y, en el plano del contexto de Río+20 puede revisarse Gonzalo Sozzo, "El principio de no regresión del derecho ambiental en el camino de la Conferencia de Rio+20" ambos publicados en "Jurisprudencia Argentina. Número Especial Derecho Ambiental”, Volume 2011-IV, Buenos Aires, Abeledo Perrot.

${ }_{18}$ Por ejemplo, en materia de agroquímicos, este principio asume un rol relevante como fundamento de la Ordenanza N ${ }^{\circ}$ 442/2012 de la localidad de Villa Mercedes en la Provincia de San Luis destinada a la Reglamentación y regulación sobre utilización, manipulación y uso de productos agroquímicos dentro del ejido municipal. Asimismo, en materia de demandas judiciales se ha hecho referencia a este principio no sólo en el caso que tratamos aquí sino, también, respecto de la reciente regulación considerada regresiva de la reglamentación de la Ley de Biocidas $N^{\circ}$ 7032/2012 de la Provincia de Chaco. 
judicial y, nuevamente, es la Corte Suprema de Justicia de Buenos Aires quien resuelve el 25 de septiembre de 2014.

$\mathrm{Al}$ resolver, la Corte alude a los argumentos de la actora que considera la segunda ordenanza regresiva en relación a los contenidos de la norma anterior, lo que sería contrario al principio de no regresión. Explica que, si bien no es la regla alegar la existencia de una infracción constitucional ante la reforma de preceptos legales, tampoco es posible desentenderse de los efectos que sobre la población podrían generar esas modificaciones en tanto se compromete el ambiente y la salud humana ${ }^{19}$.

La posible afectación de la dupla ambiente/salud, contemporáneamente se integra en un nuevo debate que posee puntos de contacto y de diferencia con el problema de los agroquímicos y que, a su vez, reedita interrogantes y desafíos: los nano-agroquímicos como herramienta para la producción agraria.

\section{Posibilidad de introducción de nano-agroquímicos. ¿Cuáles son los riesgos que comienzan a asociarse a este tipo de tecnología?}

Previo a la explicación sobre los posibles riesgos que comienza a asociarse a los nano-agroquímicos, es necesario dilucidar de forma sintética en qué consiste este tipo de tecnologías, es decir, qué es la nanotecnología.

El vocablo "nano", según el Boletín Estadístico Tecnológico (BET) publicado en junio de 2009 por el Ministerio de Ciencia, Tecnología e Innovación Productiva de la República Argentina, resalta que atañe a un prefijo del Sistema Internacional de Unidades que señala un factor de $10^{-9}$; en otras palabras, se trata de una milmillonésima parte de algo ${ }^{20}$. Es decir, que, en

${ }_{19}$ "Desentenderse de los efectos que sobre la población pueda provocar la iniciativa de reformas normativas como la aqui analizada, se exhibe, al menos en esta instancia inicial, reñido con el principio de progresividad vigente en la materia (conf. art. 4, ley 25.675; CSJN, Fallos 329:2316; esta Corte causa I. 71.446, "Fundación Biosfera", res. cit.) que, al tiempo que procura la mejora gradual de los bienes ambientales supone que los estándares de protección vigentes o actualmente logrados, no sean sustituidos por otros, inferiores u ostensiblemente ineficaces". Fragmento sentencia Picorelli, Jorge Omar y otros c/ Municipalidad de General Pueyrredón s/inconstitucionalidad Ord. N²1.296. Corte Suprema de Justicia Provincia de Buenos Aires. 25.09.2014.

${ }^{20} \mathrm{M}$. Cushen explica que las nanotecnologías involucran la manipulación de materia a una escala muy pequeña - generalmente entre 1 y 100 nanómetros-. De esta manera, "pueden explotarse nuevas propiedades y funciones que solo son posibles en la materia a dicha escala". M. Cushen , J. Kerry, M. Morris, M. Cruz Romero y E. Cummins, Nanotechnologies in the food industry, "Trends in Food Science \& Technology" 24, 30-46, 2012. Ya en 1959 en su disertación "There is a plenty of room at the bottom" el físico estadounidense Richard Feynman lo expresaba así: «Me gustaría describir un campo en el cual muy poco ha sido hecho hasta el momento, pero en el que, en principio, una gran cantidad de cosas pueden hacerse. Más aún, lo más importante es que podría tener un gran número de aplicaciones técnicas. De lo que quiero hablar es del problema de manipular y controlar objetos a muy pequeña escala». Véase: Gonzalo Bailo y Rodrigo Peiteado, Controversias que plantean las nanotecnologías en el campo jurídico. Aproximaciones desde el derecho comparado: desafios para la tutela de derechos fundamentales individuales y colectivos de incertidumbre, "XV Congreso Nacional y V Latinoamericano de Sociología Jurídica", Rosario, 2014.

Araucaria. Revista Iberoamericana de Filosofia, Política y Humanidades, año 17, nº 33. Primer semestre de 2015. Pp. 229-251. ISSN 1575-6823 e-ISSN 2340-2199 doi: 10.12795/araucaria.2015.i33.10 
términos sencillos, haciendo referencia exclusiva a escalas de medición, un nanómetro $(\mathrm{nm})$ equivale a una millonésima parte de un metro. Entonces, la nanotecnología es la capacidad técnica de estudiar y manipular la materia, con posibilidad de fabricar productos (o seres vivos) en base a materiales de escala molecular, y desarrollar diversas tecnologías a esta escala ${ }^{21}$.

Esta innovación tecnológica, según Foladori e Invernizzi, posee cuatro características relevantes que pueden ser de utilidad para identificarla. Un primer elemento lo constituye la posibilidad de construir objetos de lo más minúsculo (átomos y moléculas) a lo más grande. Un segundo elemento característico es que, a nivel atómico, no hay diferencia entre la materia biótica y la abiótica, por lo cual resulta potencialmente posible aplicar procedimientos biológicos a procesos materiales o la creación de vida artificial para desempeñar funciones específicas, entre otras. En tercer lugar, y esta es tal vez una de las características más sorprendentes, a nivel nano los elementos que conocemos convencionalmente poseen propiedades físicas diferentes. Puede que sean más resistentes, cambien de color, etcétera. Sólo para mencionar un ejemplo se puede traer a colación un elemento difundido considerablemente en el mundo científico: el nanotubo de carbono, cuya principal característica es ser más duro que el diamante y entre 50 y 100 veces más fuerte que el acero ${ }^{22}$. Una cuarta característica que puede mencionarse es que se trata de una materia pluridisciplinar, es decir, que en su seno se combinan varias tecnologías y ciencias, como la informática, la biotecnología, la tecnología de los materiales, la medicina, etcétera. Por último, a las cuatro características mencionadas por los autores, se puede agregar una quinta destacada por la organización Friends of Earth (FOE) que, luego, tendrá implicancias en relación al subtítulo de esta sección que refiere al problema de los riesgos asociados: los nano-materiales, por la escala en la que se encuentran, poseen mayor biodisponibilidad y bioactividad pudiendo ocasionarse una serie de consecuencias que, a continuación, serán explicitadas.

Tal y como lo resaltan un importante número de científicos dedicados a investigar las diversas áreas de aplicación de la nanotecnología, los posibles

\footnotetext{
${ }^{21}$ Boletín Estadístico Tecnológico, Dirección Nacional de Información Científica y Dirección nacional de Estudios, Subsecretaría de Estudios y Prospectiva, Argentina, 2009.

${ }_{22}$ "Como lo señaló el director del grupo ETC, Pat Mooney, el mundo desarrollado podría crear su propio abastecimiento de materias primas, con enormes implicaciones para las industriasy la agricultura de los países pobres. Póngase por ejemplo hipotético. Supongamos que se sustituye el acero por nanotubos de carbono. ¿Qué efectos tendría esto? Brasil exportó en 2004 un millón de toneladas de acero, lo que representó más de $18 \%$ de sus exportaciones y un monto de 334 millones de dólares. Si la sustitución de nanotubos se hiciera teniendo en cuenta sólo la resistencia del material, el millón de toneladas sería sustituido por nanotubos y reducidas las exportaciones entre 50 y 100 veces, de modo que Brasil pasaría a exportar de 10000 a 20000 toneladas, y a recibir de 3.3 a 6.7 millones de dólares". Guillermo Foladori y Noelia Invernizzi, La nanotecnología: una solución en busca de problemas, "Comercio Exterior”, Vol. 56, Núm. 4, 2006.
} 
beneficios que esta técnica parece conllevar son muy amplios y parecen no tener horizonte, ya que su uso puede ser aplicado en áreas diversas como la industria textil, la industria médica, la informática, la agroindustria, entre otras. En paralelo, también aparecen voces que alertan sobre los riegos y, en este contexto, si bien no se trata de adoptar una actitud paralizante frente al avance de la ciencia en estas innovaciones, es necesaria una perspectiva crítica constructiva que permita observar y discutir, desde un enfoque precautorio y multidisciplinario, el marco en el cual se insertan y se estudian estos descubrimientos con el objetivo de evitar situaciones de riesgo que, luego, se conviertan en irreversibles tanto para el ambiente como para la salud humana.

Dentro de los riesgos que se comienzan a asociar a las nanotecnologías, se toman como eje los discursos provenientes de algunas organizaciones no gubernamentales ${ }^{23}$ y de determinados grupos investigación ${ }^{24}$, que aportan elementos que generan una ruptura con el discurso que, de manera preponderante, circula entre los principales actores del mercado, entiéndase por ello a las empresas y los laboratorios que, muchas veces, actúan en articulación con los Estados.

Esta tecnología ha seducido también a las empresas productoras de agroquímicos debido a que las nano-partículas se caracterizan por tener mayor superficie, lo cual deriva en una mayor reactividad química, actividad biológica y comportamiento catalítico en comparación con las partículas de mayor tamaño. Los agroquímicos producidos a partir de este tipo de tecnologías podrían ser más tóxicos que los agroquímicos convencionales. Esto canalizaría una reducción de la dosis de compuesto necesaria para combatir la plaga e implicaría, a su vez, una menor residualidad y carga contaminante sobre el ambiente ${ }^{25}$. A su vez, otra característica que parecería traer grandes beneficios es que podrían encapsularse nano-partículas para que estas se activen ante determinadas condiciones. Por ejemplo, condiciones de carácter climático asociadas a la luz solar o, incluso, ante determinadas condiciones de alcalinidad que tendrían los insectos en sus estómagos al ingerir estos productos ${ }^{26}$. Por

\footnotetext{
${ }^{23}$ Un ejemplo de ello es la organización no gubernamental Friends of Earth que presiona a los gobiernos y a los actores del mercado a regular los productos de la industria nanotecnológica desde un enfoque precautorio que anteponga cuestiones de salud pública a cuestiones comerciales y a rotular productos que contengan nano-materiales. Esta organización formula denuncias y elabora informes y reportes en áreas como la alimentación, cosméticos, protectores solares, cambio climático, etcétera, promoviendo y recopilando estudios de riesgos para la salud humana y el ambiente. Pueden verse las estrategias de FOE en: http://www.foe.org/

${ }^{24}$ Un ejemplo de ello es el ETC Group que se presenta con el objetivo de "vigilar al poder, monitorear la tecnología, fortalecer la diversidad". Pueden consultarse sus trabajos en www.etcgroup.org/es

${ }^{25}$ Eder Lugo Medina, Cipriano García Gutiérrez y Redy David Ruelas Ayala,Nanotecnología y nanoencapsulación de agroquímicos, "Revista de Sociedad, Cultura y Desarrollo Sustentable", México, 2010.

${ }^{26}$ Friends of Earth, Out of the laboratory and on to our plates.Nanotechnology in food \& agriculture, 2008, Disponible en: http://libcloud.s3.amazonaws.com/93/b5/4/547/Nanotechnology in food_and_agriculture - web_resolution.pdf
} 
último, una tercera característica que resaltan las empresas y laboratorios respecto de los nano-agroquímicos es su mayor disolubilidad en ambientes acuosos así como, también, su mayor capacidad de absorción para las células, e inclusive sus núcleos, debido al tamaño que poseen las nano-partículas.

En relación a todas estas características en apariencia positivas, se advierten una serie de problemáticas referidas a la salud humana y al ambiente. $\mathrm{Si}$ bien la distinción entre salud humana y ambiente es meramente analítica dado que se trata de aspectos que son inescindibles, en referencia a la primera se puede esbozar una sintética enumeración de los principales riesgos que se han advertido: (i) El sistema de mecanismos de defensas de nuestro cuerpo no es tan efectivo para remover nano-partículas del sistema gastrointestinal así como de los demás órganos en los que se pueda almacenar como, por ejemplo, los pulmones; (ii) Las nano-partículas tienen la característica de ser más adhesivas para la superficie de nuestro cuerpo lo que produce que, junto con su tamaño tan pequeño, sean más aptas para entrar en nuestras células y tejidos; (iii) Las nano-partículas pueden resultar más tóxicas por unidad en comparación con las partículas de mayor tamaño con la misma composición química, lo que se debe principalmente a su mayor bioactividad así como a su mayor biodisponibilidad; (iv) Estudios clínicos sugieren que las nano-partículas no degradables y las pequeñas micro-partículas que, en principio, no provocan una reacción tóxica inmediata en el cuerpo luego por su mayor bioacumulabilidad pueden desembocar en las denominadas "nano-patologías", por ejemplo, granulomas, lesiones (en las células o tejidos), cáncer, coágulos de sangre, entre otros; (v) Algunos nano-materiales pueden tener la capacidad de penetrar en nuestro cuerpo a través de la piel; (vi) Existe una actual imposibilidad de parte de los científicos para predecir cuál es el máximo admisible de exposición a productos con nano-partículas, sin que se causen daños a la salud. Este último aspecto aparece como una problemática relevante sobre todo si se tiene en cuenta que existen trabajadores que manipulan estos elementos a la hora de fabricarlos. A su vez, al igual que con los agroquímicos tradicionales, se pueden presentar consecuencias perjudiciales para los trabajadores rurales que podrían manipular estos elementos antes y/o durante su aplicación en los cultivos.

Por otro lado, en relación a los riesgos asociados al ambiente, ya los agroquímicos convencionales han traído consigo una serie de dificultades como, verbigracia, daños en relación al funcionamiento de los ecosistemas así como, también, graves implicancias en términos de pérdida de biodiversidad. Debido a que los nano-agroquímicos, por las características antes mencionadas, serían más tóxicos, con mayor biodisponibilidad y bioacumulabilidad, es necesario considerar la posibilidad de que este tipo de tecnología aplicada a los agroquímicos pueda acarrear mayores problemas aún cuando se las configure para tratar de aportar soluciones. En relación a esto último, algunos científicos 
advierten que se podrían crear nuevos problemas ambientales debido a las nuevas formas de contaminación que podrían surgir de los nano-residuos a consecuencia de la producción de los nano-agroquímicos, así como también nuevas formas de contaminación en relación con las nano-partículas de agroquímicos que se liberen en el ambiente ${ }^{27}$.

A su vez, otra problemática que aparece asociada a ambos aspectos, ambiente y salud humana, es la posibilidad de que las nano-partículas, al ser liberadas en el ambiente con el objetivo de combatir plagas, se constituyan luego como causa de problemas en la cadena trófica, ya que en relación a este punto existe una considerable laguna en el conocimiento ${ }^{28}$. Además, desde el ámbito científico también se advierte que las especies podrían absorber las nano-partículas liberadas en el ambiente, lo que traería como consecuencia que se produzcan modificaciones en el ADN de plantas o animales. Esto podría provocar situaciones impensadas, más aún si se tiene en cuenta que, luego, muchas de esas plantas o animales pueden ser ingeridos por el ser humano trayendo como consecuencia una larga cadena de modificaciones cuyas consecuencias son desconocidas.

Esta última posición es tal vez la más crítica sobre la cuestión, sin embargo, aún si se asumiera una posición más laxa, debido al desconocimiento que se visibiliza sobre las consecuencias potencialmente negativas, parece necesario construir canales para decidir de la manera más participativa y colectiva posible sobre la libre circulación de estos productos hasta tanto se conozcan fehacientemente qué consecuencias pueden provocar y teniendo en consideración el derecho humano a un ambiente sano y la necesidad de tutela de la naturaleza.

Entonces, y a modo de síntesis, puede afirmarse que las nano-partículas implicarían nuevos riesgos ya que son químicamente más reactivas, tienen mayor y mejor acceso a nuestros cuerpos, poseen mejor bioacumulabilidad y más bioactividad para introducir nuevos riesgos de toxicidad, y pueden comprometer nuestro sistema inmune. Asimismo, debido a su acumulabilidad es que, en un principio, puede que no provoquen efectos en el ambiente o en la salud pero en el mediano o largo plazo no se tiene un conocimiento certero sobre su inocuidad.

Siguiendo con la síntesis, se debe recordar que no se posee un conocimiento certero sobre qué nivel de exposición a nano-partículas no generaría un daño, o bien, si realmente existe un nivel seguro de exposición para la salud teniendo en cuenta sus características técnicas y específicas.

\footnotetext{
27 Para mayor información sobre el problema de los nano-residuos: Gonzalo Bailo, Estrategias jurídicas locales para el gobierno de los nanoresiduos. Construyendo una agendas regulatoria para nanomateriales en el derecho argentino, "Revista de Derecho Ambiental No 38", Buenos Aires, Abeledo Perrot, 2014.

${ }^{28}$ La cadena trófica es el proceso de transferencias nutritivas de una especie a otra dentro de una comunidad biológica, en donde el uno se alimenta del precedente y es alimento a la vez del siguiente.
} 
Este estado de incerteza sobre diversos aspectos de las nano-tecnologías, y de los nano-agroquímicos en particular, requiere colocar el foco de atención sobre los mecanismos para la toma de decisiones y las regulaciones que sería necesario construir, sobre todo, teniendo en cuenta que algunos científicos han advertido que ya se encuentran productos con nano-partículas en el mercado bajo el nombre de microemulsiones como, por ejemplo, el caso del Primo MAXX de Syngenta ${ }^{29}$ respecto del cual, miembros de Syngenta Australia, al momento de ser consultados por Friends of Earth, dejaron entrever que contiene partículas de $100 \mathrm{~nm}^{30}$.

\section{Agendas abiertas de construcción jurídico-institucional: algunos desafíos en la intersección entre protección de los derechos humanos y nuevas tecnologías}

En el momento actual existe una proliferación de riesgos que se asocian a nuevas tecnologías y cuyas construcciones sociales en tanto riesgos se replican, aunque de heterogénea manera, en diferentes circunstancias socio-geográficas. La expansión del uso los agroquímicos y, aún más cerca en el tiempo, los adelantos en materia de nano-agroquímicos constituyen algunas de las hipótesis que plantean importantes desafíos.

En el supuesto de los agroquímicos confluyen una serie de normativas que conjugan la autorización de comercialización de los productos y su uso en el territorio, así como algunas regulaciones que de manera tangencial afectan este tipo de práctica. Con los años han proliferado una serie de demandas judiciales en las que se denuncian fumigaciones ilegales o se discute sobre la constitucionalidad de normas locales más o menos protectorias. Este proceso de judicialización del problema permite visualizar una serie de actores sociales que bregan por una mayor tutela del ambiente y de la salud y que requieren de espacios más plurales para la toma de decisiones. Luego de varios años, desde diferentes instancias estatales, se va colocando el acento en las consecuencias ambientales y sociales que generó, y sigue generando, este modelo de producción agraria $\mathrm{y}$, aunque de manera incipiente, diversos actores sociales

\footnotetext{
${ }^{29}$ Esta empresa es la mayor y más grande productora de agroquímicos en el mundo. Si se desea saber más acerca de la composición de este regulador de crecimiento vegetal Primo MAXX consultar en: http://www.syngentaprofessionalproducts.com/prodrender/index.aspx?prodid=747

${ }^{30}$ Lo expuesto permite mencionar una discusión también vigente acerca de qué elementos se consideran dentro de los parámetros nanos y cuáles no. En este sentido, existe una puja entre las empresas, los laboratorios, los Estados en algunas ocasiones, y las organizaciones no gubernamentales debido a que no existe acuerdo sobre cuál es la frontera para designar a un producto como nanotecnológico. Ello obviamente se debe a que si es considerado nanotecnológico le resultaría aplicable una serie de reglamentaciones y programas regulatorios distintos de los materiales convencionales que comienzan a aparecer aunque de modo incipiente en algunos países y, en este sentido, las empresas y laboratorios abogan por parámetros más laxos para considerar a un elemento dentro del mundo nano.
} 
han tomado nota de variadas lecciones de las cuales aprender. Entre ellas, la necesidad de conocer más profunda y acabadamente sobre las consecuencias perniciosas que acarrean estos agentes una vez liberados al medio así como, también, la necesaria construcción de espacios participativos para la toma de decisiones sobre riesgos colectivos.

Este camino se sigue transitando y los avances jurídico-institucionales no son rápidos; por el contrario, muchas veces se articulan con respuestas a movilizaciones sociales de envergadura o bien con decisiones judiciales que han ocupado un espacio central en el debate público. Como respuesta ante demandas que llegan a construirse con la suficiente potencia, aparecen algunas, aunque escasas, innovaciones institucionales como la generación de comisiones para la investigación de los efectos de los agroquímicos en la salud o el seguimiento epidemiológico de parte de las agencias de salud estatales.

En este tránsito, hoy aparece la posibilidad de aplicar al agro los nanoagroquímicos y se recrea así una instancia en que se deben tomar decisiones sobre un agente cuyas consecuencias son controvertidas y que podrían repercutir negativamente en la protección del derecho humano a un ambiente sano. Se renuevan entonces los desafíos. Una mirada precautoria sobre el tema implicaría generar las condiciones jurídico-institucionales para conocer de manera más acabada sobre este agente considerando no sólo los actores del sistema científico sino, también, asignando espacio a otras voces que ya vienen alertando sobre este tema. Asimismo, esa mirada conlleva la necesidad de generar espacios públicos de discusión y de toma de decisiones sobre la autorización de comercialización y aplicación de este tipo de sustancias.

En esta tarea, un aporte posible desde el derecho, en su rol no sólo regulatorio sino de diseño institucional, estaría dado por la posibilidad de repensar regulaciones y mecanismos de toma de decisiones que han sido inspirados por anteriores paradigmas en los que, desde las ciencias, se podían construir informaciones sobre las cuales decidir. Hoy en día, el principio de precaución implica, por una parte, profundizar en las investigaciones ante este tipo de hipótesis y, además, dialogar con diferentes espacios de producción de saberes. Por otra parte, este principio conduce al diseño de mecanismos para la toma de decisiones por parte de diversos actores sociales concernidos entre los cuales se encuentran las agencias estatales pero, también, organizaciones no gubernamentales, afectados, etcétera.

Las actuales regulaciones jurídicas y mecanismos para decidir sobre riesgos controvertidos en general poseen escasa plasticidad para, por ejemplo, atender la multiplicidad de aristas que conlleva la problemática de la nanotecnología en general y de los nano-agroquímicos en particular. Ello no es sino una manifestación de la necesidad de sortear los parámetros del derecho moderno y construir desde lo inestable. En estos casos no existe certeza al 
interior del campo científico ni, más allá del mismo, sobre los riesgos que su expansión y aplicación pueden acarrear. Esto podría ser visualizado como una crisis aunque, también, puede constituirse como una oportunidad para, analizando la experiencia ya transitada sobre agroquímicos, tomar elementos para gestionar de manera más idónea el problema que comienza a presentarse ante la aplicación de nano-agroquímicos a la producción agraria. En esta tarea, la tutela de los derechos humanos ha de constituirse como un elemento fundamental para inspirar los diseños jurídico-institucionales necesarios para atender es tema así como otras hipótesis de nuevas tecnologías con las que las sociedades conviven. 


\section{Referencias bibliográficas}

Gonzalo Bailo, Estrategias jurídicas locales para el gobierno de los nanoresiduos. Construyendo una agenda regulatoria para nanomateriales en el derecho argentino, Revista de Derecho Ambiental $N^{\circ} 36$, Abeledo Perrot, Buenos Aires, 2014.

Gonzalo Bailo y Rodrigo Peiteado, Controversias que plantean las nanotecnologías en el campo jurídico. Aproximaciones desde el derecho comparado: desafios para la tutela de derechos fundamentales individuales y colectivos de incertidumbre, "XV Congreso Nacional y V Latinoamericano de Sociología Jurídica", Rosario, 2014.

Ulrich Beck, La sociedad del riesgo mundial, Paidos, Barcelona, 2007.

Ulrich Beck, La sociedad del riesgo global, Barcelona, Siglo XXI, 2002.

Ulrich Beck, La sociedad del riesgo. Hacia una nueva modernidad, Barcelona, Paidos, 1998.

Ulrich Beck y Christoph Lau,Second modernity and research agenda: theoretical and empirical explorations in the "meta-change" of modern society, "The British Journal of sociology", Volume 56. Number 4, 2005.

María Valeria Berros, Construyendo el principio de no regresión en el derecho argentino, "Jurisprudencia Argentina. Número Especial Derecho Ambiental" Volume 2011-IV, Buenos Aires, Abeledo Perrot.

M. Cushen , J. Kerry, M. Morris, M. Cruz Romero y E. Cummins, Nanotechnologies in the food industry, "Trends in Food Science \& Technology" 24, 30-46, 2012.

José Esteve Pardo, El desconcierto del Leviatán. Política y derecho ante las incertidumbres de la ciencia, Madrid, Marcial Pons, 2009.

José Esteve Pardo, Técnica, riesgo y derecho. Tratamiento del riesgo tecnológico en el Derecho Ambiental, Barcelona, Ariel, 1999.

Guillermo Foladori y Noelia Invernizzi, La nanotecnología: una solución en busca de problemas, "Comercio Exterior", Vol. 56, Núm. 4, 2006.

Eder Lugo Medina, Cipriano García Gutiérrez y Redy David Ruelas Ayala, Nanotecnología y nanoencapsulación de agroquímicos, "Revista de Sociedad, Cultura y Desarrollo Sustentable", México, 2010.

Christine Noiville, Du bon gouvernement des risques. Le droit et la question $d u$ «risque acceptable », Paris, Presses Universitaires de France, 2003.

Dominique Pestre, Introduction aux sciences studies, Paris, La Découverte, 2006.

Michel Prieur y Gonzalo Sozzo (Coord.) La non régression en droit de l'environnement, Bruxelles, Bruylant, 2012.

Boaventura de Sousa Santos, Crítica de La razón indolente. Contra el desperdicio de la experiencia, Bilbao, Editorial Desclée de Brouwer, 2003. 
Boaventura de Sousa Santos, Um discurso sobre as ciências, Porto, Ediciones Afrontamento, 2002.

ETC Group, Nanotech's "Second Nature” Patents: Implications for the Global South.Ottawa: ETC Group, 2005

ETC Group,Let's Look Before We Leap: Civil society calls for TechnologyAssessment as part of any Copenhagen deal, 2009.

Medina, Eder Lugo; García Gutiérrez, Cipriano; Ruelas Ayala, Rey David, Nanotecnología y nanoencapsulación de agroquímicos, Revista de Sociedad, Cultura y Desarrollo Sustentable, México, 2010.

Silvia Ribeiro, Nanotecnología: del campo a su estómago, Catedra UNESCO en Tecnología, Desenvolupament Sostenible, Desequilibris i Canvi Global, Observatorio de la Deuda en la Globalización, Univesitat Politecnica de Catalunya, España, 2004.

Gonzalo Sozzo, "El principio de no regresión del derecho ambiental en el camino de la Conferencia de Rio+20", "Jurisprudencia Argentina. Número Especial Derecho Ambiental" Volume 2011-IV, Buenos Aires, Abeledo Perrot.

\section{Reportes e informes oficiales y de organizaciones no gubernamentales}

Ministerio de Ciencia, Tecnología e Innovación Productiva, Boletín estadístico tecnológico $N^{\circ} 3,2009$.

Friends of Earth,Nanotechnology, climate and energy: Over-heated promises and hot air?U.S Edition, 2010, Disponible en:http://www.foe.org.

Friends of Earth, Report out of the laboratory and on to our plates. Nanotechnology in Food \& Agriculture, 2008, Disponible en: http:// libcloud.s3.amazonaws.com/93/b5/4/547/Nanotechnology in food and agriculture - web resolution.pdf 
